\title{
Androgen excess and diagnostic steroid biomarkers for nonclassic 21-hydroxylase deficiency without cosyntropin stimulation
}

 Richard J Auchus ${ }^{1,4}$ and Deborah P Merke ${ }^{2,5}$}

${ }^{1}$ Division of Metabolism, Endocrinology and Diabetes, University of Michigan, Ann Arbor, Michigan, USA, ${ }^{2}$ National Institutes of Health (NIH) Clinical Center, Bethesda, Maryland, USA, ${ }^{3}$ School of Public Health, ${ }^{4}$ Department of Pharmacology, University of Michigan, Ann Arbor, Michigan, USA, and ${ }^{5}$ Eunice Kennedy Shriver National Institute of Child Health and Human Development, Bethesda, Maryland, USA

Correspondence should be addressed to A F Turcu

Email

aturcu@umich.edu

The authors apologise for an error in Table 2 of the above article published in the July 2020 issue (vol 183 Iss 1 pp 63-71) of the European Journal of Endocrinology. The values for 17OHP4 should be as given in the table below and not as published. The correct Table 2 is published in full below.

Table 2 Comparison of steroid concentrations between patients with nonclassic 21-hydroxylase deficiency (N21OHD) and controls. Data are expressed as median (interquartile range).

\begin{tabular}{|c|c|}
\hline Steroid $(\mathrm{nmol} / \mathrm{L})$ & N210HD $(n=32)$ \\
\hline 17OHP4 & $18.1(8.7-33.0)$ \\
\hline 16OHP4 & $2.4(1.4-4.9)$ \\
\hline $21 \mathrm{dF}$ & $1.8(1.3-5.3)$ \\
\hline Progesterone & $0.8(0.5-1.4)$ \\
\hline Cortisol & $202.5(125.9-442.8)$ \\
\hline Cortisone & $53.7(30.5-72.3)$ \\
\hline Corticosterone & $1.6(0.8-3.5)$ \\
\hline $11 \mathrm{dF}$ & $0.8(0.5-1.4)$ \\
\hline DOC & $0.3(0.3-0.4)$ \\
\hline A4 & $4.3(2.0-8.1)$ \\
\hline Testosterone & $1.1(0.5-2.7)$ \\
\hline 110HA4 & $9.6(5.1-25.9)$ \\
\hline $11 \mathrm{KA} 4$ & $1.1(0.7-1.7)$ \\
\hline $110 \mathrm{HT}$ & $1.0(0.3-1.7)$ \\
\hline $11 \mathrm{KT}$ & $1.8(1.2-3.3)$ \\
\hline $11 \mathrm{KT} / \mathrm{Testosterone}$ & $1.8(1.2-3.0)$ \\
\hline $110 \mathrm{HA} 4 / \mathrm{A} 4$ & $2.6(2.1-4.6)$ \\
\hline A4/testosterone & $3.8(2.8-4.5)$ \\
\hline
\end{tabular}

\begin{tabular}{c}
\hline Controls $(n=54)$ \\
\hline $2.8(0.9-4.3)$ \\
$0.6(0.4-0.9)$ \\
$0.0(0.0-0.3)$ \\
$0.4(0.3-0.8)$ \\
$249.1(208.9-373.7)$ \\
$51.5(40.3-60.8)$ \\
$5.9(3.5-13.6)$ \\
$0.9(0.4-1.6)$ \\
$0.5(0.4-0.9)$ \\
$3.9(2.2-8.5)$ \\
$1.6(0.8-4.3)$ \\
$4.5(2.9-7.1)$ \\
$0.6(0.4-0.8)$ \\
$0.4(0.3-0.6)$ \\
$0.9(0.6-1.3)$ \\
$0.6(0.2-0.9)$ \\
$1.2(0.7-2.1)$ \\
$3.5(2.4-4.2)$ \\
\hline
\end{tabular}

\begin{tabular}{c}
\hline Ratio of medians N210HD/controls \\
\hline 6.5 \\
4.1 \\
N/A \\
1.8 \\
0.8 \\
1.0 \\
0.3 \\
0.8 \\
0.6 \\
1.1 \\
0.7 \\
2.1 \\
1.7 \\
2.2 \\
2.0 \\
3.3 \\
2.0 \\
1.3
\end{tabular}

\begin{tabular}{c}
\hline $\boldsymbol{P}$ \\
\hline$<0.0001$ \\
$<0.0001$ \\
$<0.0001$ \\
0.0018 \\
0.197 \\
0.855 \\
$<0.0001$ \\
0.626 \\
0.113 \\
0.798 \\
0.195 \\
$<0.0001$ \\
0.0001 \\
0.0019 \\
$<0.0001$ \\
$<0.0001$ \\
$<0.0001$ \\
0.274
\end{tabular}

A4, androstenedione; DOC, 11-deoxycorticosterone; 17OHP4, 17 $\alpha$-hydroxyprogesterone; 16OHP4, 16 $\alpha$-hydroxyprogesterone; 21dF, 21-deoxycortisol; $11 \mathrm{dF}$, 11-deoxycortisol; 11OHA4, 11 $\beta$-hydroxyandrostenedione; 11 KA4, 11-ketoandrostenedione; $11 \mathrm{OHT} 11 \beta$-hydroxytestosterone; $11 \mathrm{KT}$, 11-ketotestosterone. 
Downloaded from Bioscientifica.com at $04 / 26 / 2023$ 11:32:45AM

via free access 